\title{
Questes
}

vestes Revue pluridisciplinaire d'études médiévales

27 | 2014

Naissances

\section{Génération et création poétique : la naissance d'une femme écrivain}

Barbara Falleiros

\section{(2) OpenEdition}

Journals

Édition électronique

URL : http://journals.openedition.org/questes/774

DOI : 10.4000 /questes.774

ISSN : 2109-9472

Éditeur

Les Amis de Questes

Édition imprimée

Date de publication : 15 janvier 2014

Pagination : 89-108

ISSN : 2102-7188

\section{Référence électronique}

Barbara Falleiros, « Génération et création poétique : la naissance d'une femme écrivain », Questes [En ligne], 27 | 2014, mis en ligne le 15 janvier 2014, consulté le 01 mai 2019. URL : http:// journals.openedition.org/questes/774; DOI : 10.4000/questes.774 


\title{
Génération et création poétique : la naissance d'une femme écrivain ${ }^{1}$
}

\author{
Barbara FALLEIROS \\ Université Paris-Sorbonne (Paris IV)
}

La littérature des derniers siècles du Moyen Âge se caractérise par l'émergence d'une conscience d'auteur et le développement de réflexions approfondies sur le fait littéraire ${ }^{2}$. Dans ce contexte, les citations ou les réécritures de récits mythologiques - des fables ovidiennes, notamment servent au développement d'un discours métaphorique sur la création poétique. Chez Christine de Pizan, l'auteur qui nous occupera principalement ici, «la fable [devient] un instrument d'investigation du moi $^{3} »$, mais ce moi poétique porte la marque d'une irréductible altérité : Christine se distingue des autres poètes par le fait d'être une femme. Or, dans son utilisation de mythes et de métaphores pour penser l'écriture et pour s'affirmer en tant qu'auteur, Christine engage très souvent sa condition féminine. Dans cet article, nous étudierons trois œuvres allégoriques de l'auteur, Le Livre de la mutacion de Fortune, Le Livre de la

\footnotetext{
${ }^{1}$ Le présent article s'inscrit dans le cadre d'un projet de recherche soutenu par l'attribution d'une allocation Institut Émilie du Châtelet (IEC) - Région Île-deFrance.

${ }^{2}$ Voir, par exemple, à ce sujet : Michel Zink, La subjectivité littéraire. Autour du siècle de saint Louis, Paris, PUF, coll. «Écriture », 1985 ; Jacqueline Cerquiglini-Toulet, "Un engin si soutil». Guillaume de Machaut et l'écriture au XIV siècle, Paris, Champion, coll. "Bibliothèque du XV siècle», 1985 ; Didier Lechat, Dire par fiction. Métamorphoses du "je" chez Guillaume de Machaut, Jean Froissart et Christine de Pizan, Paris, Champion, coll. « Etudes christiniennes », n 7, 2005.

${ }^{3}$ Jean-Claude Mühlethaler, « Entre amour et politique : métamorphoses ovidiennes à la fin du Moyen Âge », Cahiers de recherches médiévales [En ligne], 9, 2002, mis en ligne le 04 septembre 2007, http://crm.revues.org/76, § 36 (page consultée le 7 juillet 2013).
} 
cité des dames et Le Livre de l'advision Cristine, et montrerons comment la poétesse explore la métaphore de l'écriture-génération-naissance dans une perspective féminine.

Pour mieux rendre compte de la singularité de la démarche de Christine de Pizan, il serait intéressant d'observer, dans un premier temps, le développement de métaphores analogues dans l'œuvre d'un autre grand auteur de cette période, Guillaume de Machaut ${ }^{5}$. Son Voir dit raconte une histoire d'amour qui se vit par l'écrit, au travers de lettres échangées entre le vieux poète et une jeune fille. À un moment de la narration où des soupçons d'infidélité pèsent sur la dame, l'auteur introduit la question complexe des liens entre mensonge et vérité. Dans un rêve, le portrait de la dame rappelle au narrateur la fable ovidienne du corbeau et de la corneille ${ }^{6}$. « Tous voirs ne sont pas biaux a dire », disait la corneille ; parfois, mieux vaut ne pas connaître la vérité. Or, la fable racontée par le portrait renferme deux histoires de naissances singulières. La première est celle d'Érichthonios, un être mi-homme, mi-serpent, fruit du désir ardent de Vulcain pour Pallas. Érichthonios était né de la terre sur laquelle le dieu avait répandu sa semence : prolem sine mater creatam, écrit Ovide, un enfant sans mère ${ }^{7}$. La deuxième naissance est celle d'Esculape, une

${ }^{4}$ Nos éditions de référence seront les suivantes: Christine de Pizan, Le Livre de la mutacion de Fortune, éd. Suzanne Solente, Paris, A \& J Picard, coll. «Société des anciens textes français », $\mathrm{n}^{\circ} 85,1959,4$ vol. ; Christine de Pizan, La città delle dame, a cura di Patrizia Caraffi, edizione di Earl Jeffrey Richards, Milano, Luni Editrice, coll. « Biblioteca medievale », n² 2, 1997 ; Christine de Pizan, Le Livre de l'advision Cristine, éd. Liliane Dulac et Christine Reno, Paris, Champion, coll. «Études christiniennes $", \mathrm{n}^{\circ} 4,2001$.

${ }^{5}$ Guillaume de Machaut, Le Livre du voir dit, éd. et trad. Paul Imbs, introduction et révision par Jacqueline Cerquiglini-Toulet, Paris, Librairie Générale Française, coll. « Lettres gothiques », 1999.

${ }^{6}$ Ibid., v. 7665-8106 («Comment le portrait de Toute-Belle se plaint à l'amant »), p. $682-708$.

${ }^{7}$ Ibid., v. 7817-7835, p. 692. L'auteur de l'Ovide moralisé traduit ainsi ce passage: «Un enfant, qui double forme a,/Que l'en claimme Eritoniun, /Nasqui de cele emission. /Ce fu merveilles, quant sans mere /Fu nez de la semence au pere " (Ovide moralisé : poème du commencement du XIV siècle, éd. Cornelis de Boer, Martina 
naissance par césarienne, survenue au moment où sa mère, Coronis, décédait des suites des blessures que Phébus lui avait infligées en la transperçant d'une flèche, après avoir découvert son infidélité ${ }^{8}$.

Quel est le sens de ce jeu d'enchâssements narratifs renfermant des histoires de naissances sans mère ? Machaut y mène une réflexion sur la genèse de l'écriture, sur la forme et la matière poétiques. De Nature, le poète avait reçu «sens, retorique et musique », liés aux aspects formels de l'écriture. L'amour en fournit la matière : on ne peut pas écrire sans être amoureux. Mais que se passe-t-il si cet amour n'est pas, ou n'est plus, partagé ? On a fait remarquer qu'à la différence de Narcisse, amoureux de son propre reflet, « le désir de l'autre existe chez le poète, mais la réalité de l'amour, de la part de l'autre, n'est pas nécessaire à la création. ». L'écriture naît ainsi du seul désir, « comme Érichthonios s'était engendré du seul désir de Vulcain, comme Esculape était né de la seule volonté de Phoebus ${ }^{9}$. ।

À l'instar de ses contemporains, Machaut subit l'influence du Roman de la Rose de Jean de Meun, où les récits de naissances mythiques et la métaphore écriture-procréation signifient les liens entre l'art poétique et la nature $^{10}$. Une cinquantaine d'années plus tard, c'est au tour de Christine de

G. de Boer et Jeannette T. M. van't Sant, Vaduz, Sändig, H. R. Wohlwend, 1988 [Fac-sim. de l'éd. de 1915-1938], t. I, Second Livre, v. 2236-2240, p. 220.

${ }^{8}$ Guillaume de Machaut, Le Livre du voir dit, op. cit., v. 7989-8032, p. 702-704.

${ }^{9}$ Jacqueline Cerquiglini-Toulet, «Un engin si soutil »..., op. cit., p. 154.

${ }^{10}$ Dans le Roman de la Rose, l'image de Nature qui façonne dans sa forge des êtres humains, le mythe de Pygmalion ou encore le discours de Genius se construisent autour d'un réseau métaphorique qui associe l'acte sexuel, les travaux de l'écrivain, $\mathrm{du}$ laboureur et du forgeron. Voir Pierre-Yves Badel, Le Roman de la Rose au $X I V^{e}$ siècle : étude de la réception de l'œuvre, Genève, Droz, 1980, p. 54. Comme l'écrit encore Didier Lechat au sujet du discours de Genius : «Le prêtre de Nature, qui a compétence pour tout ce qui relève de la génération et de la reproduction, unit dans un même faisceau métaphorique l'usage du stylet sur les tablettes, celui du marteau sur l'enclume et celui des socs de la charrue dans la terre : équivoques filées entre les activités créatrices de l'homme et l'acte de procréation » (Didier Lechat, Dire par fiction : métamorphoses du je chez Guillaume de Machaut, Jean Froissart et Christine de Pizan, Paris, Champion, 2005, p. 385). 
Pizan de penser l'écriture et le moi poétique par le biais d'une série d'images de génération, d'engendrement et de naissance. Chez Christine, nous assistons néanmoins à un renouvellement de la matière poétique, qui échappe désormais à la lyrique amoureuse. Dès le début $\mathrm{du} \mathrm{Xv}^{\mathrm{e}}$ siècle, l'auteur abandonne la composition de dits amoureux et se tourne vers des sujets politiques, moraux et historiques. C'est pourtant dans ces types de traités, comme l'a bien montré Didier Lechat, que l'on remarque le plus fort investissement $\mathrm{du}$ « je », le recours continuel au songe, à l'allégorie et à la mythologie.

Il faut dire que chez Christine, le problème de l'autorité de l'écrivain est un enjeu primordial : pour faire face à l'univers masculin du discours clérical, l'auteur doit en effet se construire un portrait de "clergesse », détentrice d'un savoir accrédité. Puisque toute tentative de voiler son identité féminine s'avérerait inutile, il lui faut au contraire toujours l'assurer, l'excuser, la justifier :

[...] dans toute grande œuvre, le sujet d'énonciation s'élabore par l'intersubjectivité. Mais les femmes-auteurs ressentent peut-être plus fortement cette nécessité esthético-philosophique - malheur fait chance - parce que l'expérience de l'altérité leur est imposée par la société : c'est le point de départ de la réflexion de l'écrivain Christine de Pizan ${ }^{11}$.

Christine, qui n'a pas d'emblée l'autorité du clerc pour présenter, tout comme Machaut, une écriture «conçue toute seule», substitue aux naissances sans mère la maternité et les filiations symboliques. Les solutions qu'elle propose évoluent pourtant au fil de ses œuvres vers

${ }^{11}$ Michèle Weil, " "Je suis comme toy": dialogie de Christine de Pizan », dans Une femme de lettres au Moyen Âge. Études autour de Christine de Pizan, éd. Liliane Dulac et Bernard Ribémont, Orléans, Paradigme, coll. « Medievalia », n 16, 1995, p. $373-381$, p. 373. 
l'affirmation de plus en plus sûre de la possibilité d'écriture par une femme.

\section{La Mutacion de Fortune : renaître dans un corps d'homme}

Composé au début de la période la plus prolifique de la carrière de Christine, Le Livre de la mutacion de Fortune (1400-1403) est, par sa matière et son étendue -24000 vers environ -, la fiction poétique la plus ambitieuse de l'auteur. Le récit biographique allégorisé renferme un long exposé d'histoire du monde, une histoire peinte sur les murs du château de Fortune, visité par la narratrice. Dans ce texte, il est question en effet de l'emprise qu'exerce sur les hommes Fortune, puissance instable et imprévisible, qui décide de l'essor ou de la décadence de peuples, d'empires et d'individus.

Pour Christine, l'« enarracion de ses aventures » est l'occasion de signifier sa renaissance, de remémorer les circonstances de sa mutation de femme-épouse en écrivain. Le problème de l'identité, qui implique bien évidemment une quête de reconnaissance littéraire, d'immortalisation du nom par l'écrit, se pose dès le prologue où la narratrice annonce de sa métamorphose par l'artifice rhétorique de présentation de l'auteur. On y reconnaît l'influence des fables mythologiques d'Ovide :

Vous diray qui je suis, qui parle,

Qui de femelle devins male

Par Fortune, qu'ainsy le voult ;

Si me mua et corps et voult

En homme naturel parfaict;

Et jadis fus femme, de fait

Homme suis, je ne mens pas ${ }^{12}$.

Si Christine insiste à plusieurs reprises sur la véracité de son récit, jouant sur la célèbre rime songe-mensonge du Roman de la Rose, elle entend fournir les clés d'interprétation de l'allégorie dont elle maîtrise les

${ }^{12}$ Christine de Pizan, Le Livre de la mutacion..., éd. cit., t. I, v. 141-147, p. 12. 
procédés. La métamorphose est bien évidemment fictive, son récit est le résultat d'un travail poétique, mais ce n'est qu'une manière plus recherchée de dire la vérité : «[...] soubz figure de metaphore, c'est a dire de parole couverte, sont muciees maintes secretes sciences et pures verite ${ }^{13} »$, dira-t-elle plus tard dans l'Advision. La poésie est une parole vraie : «Et si n'est mençonge, ne fable, /A parler selon methafore, /Qui pas ne met verité fore $^{14}$. »

C'est donc par métaphore que la poétesse raconte son origine dans la Mutacion. On s'attendrait à la voir établir une filiation entièrement allégorique; pourtant, sa première description est celle de la figure historique de son père, Thomas de Pizan. Ce père lettré, médecin et astrologue, s'impose dès lors comme une figure d'autorité sur laquelle Christine assoit sa légitimité intellectuelle. La filiation s'avère hautement symbolique. La narratrice se décrit comme étant le portrait de son père, à un détail près: «De toutes choses mon pere/Bien ressemblay et proprement, /Fors du sexe tant seulement ${ }^{15} »$. Le sexe féminin est un trait qu'elle tient de sa mère, Nature, cette mère qui décida délibérément des caractéristiques de l'enfant et dont le désir d'avoir « une femelle a elle ressemblable ${ }^{16} »$ ne l'empêcha pas de doter sa fille des attributs physiques et intellectuels du père.

Cette réflexion étonnante sur les caractéristiques innées n'est pas anodine. En admettant qu'une femme puisse naître avec les mêmes facultés raisonnables qu'un homme, Christine de Pizan touche à un épineux problème ontologique. Comme l'a démontré Jeffrey Richards, ce postulat répond aux conceptions scolastico-thomistes de l'homme comme un être créé essentiellement pour penser et concevoir, tandis que la femme n'aurait

\footnotetext{
${ }^{13}$ Christine de Pizan, Le Livre de l'advision..., éd. cit., partie I, chap. I, p. 3.

${ }^{14}$ Christine de Pizan, Le Livre de la mutacion..., éd. cit., t. I, v. 1032-1034, p. 41.

${ }^{15}$ Ibid., t. I, v. 394-396, p. 20.

${ }^{16}$ Ibid., v. 391.
} 
été créée que pour enfanter ${ }^{17}$. Ce sont les pratiques culturelles qui empêchent l'accès des femmes au savoir, et non l'imperfection ou l'inachèvement de leur être - idée que Christine dénoncera d'ailleurs fréquemment dans ses œuvres : «Se droit regnoit, riens n’y perdroit/La femmelle, ne que le filz, /Mais, en mains lieux, [...]/Regnent plus coustumes que drois ${ }^{18}$.»

Revenons à la mère de la narratrice. Cette femme puissante, qui l'allaita et la nourrit, n'est autre que Nature elle-même. La personnification est certes inspirée de la figure que Jean de Meun emprunta au De planctu Naturae d'Alain de Lille, cependant, la morale naturelle que Nature incarnait dans ces textes ne se situe plus, chez Christine, dans le domaine de la sexualité ${ }^{19}$. La Nature du Roman de la Rose était, en effet, une figure de l'engendrement, de la procréation d'un point de vue masculin. Christine, au contraire, insiste sur la représentation de la maternité, image qu'elle développera dans ses œuvres postérieures. De même, l'art de Nature s'est subtilement transformé, du Roman de la Rose à la Mutacion de Fortune : il ne s'agit plus du travail au marteau, dans la forge, mais de la fabrique de bijoux dans une orfèvrerie. À l'occasion du mariage de sa fille Christine, Nature lui offre un chapelet serti de pierres précieuses appelées « discrecion », « consideracion », " retentive » et "memoire ». Comme Machaut à qui Nature avait procuré les outils formels de l'écriture, ces dons que Christine reçoit à sa naissance et en grandissant, à savoir la faculté intellectuelle et le goût du savoir, la prédisposent à écrire. Encore faut-il en trouver la matière ; c'est Fortune qui se charge de la lui fournir :

\footnotetext{
${ }^{17}$ Voir Earl Jeffrey Richards, «Rejecting essentialism and Gendered Writing: The Case of Christine de Pizan ", dans Gender and Texts in the Later Middle Ages, éd. Jane Chance, Gainesville, University of Florida Press, 1996, p. 96-131, p. 97.

${ }^{18}$ Christine de Pizan, Le Livre de la mutacion..., éd. cit., t. I, v. 417-423, p. 21.

${ }^{19}$ «[...] il n'y a pas de morale naturelle chez Jean de Meun hors du champ de la sexualité », Pierre-Yves Badel, Le Roman de la Rose au XIV siècle. Étude de la réception de l'œuvre, Genève, Droz, 1980, p. 50, n. 112.
} 
« Tant m'est Fortune apportant /De ses mes, que matiere ay /D'en parler », dit la narratrice, et plus loin, «[...] par quoy devint, /Mon sens plus soubtil $\operatorname{assez}^{20}$. »

Les épisodes de la biographie de Christine de Pizan, les revers de fortune qui précèdent et préparent son entrée dans la vie littéraire, sont bien connus : quelques années après avoir perdu son père, Christine se retrouve veuve, à vint-cinq ans, avec trois enfants à charge, cherchant dans l'écriture un moyen de subsistance et de consolation. Dans la Mutacion de Fortune, la poétesse se remémore les circonstances tragiques de la disparition de son époux, événement qu'elle évoque sur le mode allégorique comme le passage d'une violente tempête maritime qui emporte le conducteur de sa nef. La souffrance de la narratrice est si profonde que Fortune elle-même s'apitoie sur son sort et opère sa métamorphose physique :

Une foiz toute recassee

Demouray, com toute chose transsie

M'endormi a une ressie ;

Adont vers moi vint ma maistresse,

Qui a plusieurs la joye estrece,

Si me toucha par tout le corps

Chacun membre, bien m'en recors,

Manÿa et tint a ses mains

Puis s'en alla et je me remains ${ }^{21}$.

À son réveil, Christine se sent «transmuee »; ses membres sont plus forts, sa voix est devenue plus grave : elle peut désormais se faire entendre. Notons que la transformation n'est pas strictement corporelle, mais s'accompagne d'une nouvelle force de caractère: «Car tout soubdainement mua/Celle grant paour et la doubte/Ou je me confondoie toute $^{22} »$. Avec une vigueur renouvelée, la narratrice peut prendre le contrôle de la nef et la réparer :

\footnotetext{
${ }^{20}$ Christine de Pizan, Le Livre de la mutacion..., éd. cit., t. I, v. 30-32 et v. 38-39, p. 8.

${ }^{21}$ Ibid., v. 1322-1330, p. 51.

${ }^{22}$ Ibid., v. 1344-1346.
} 
Quant ainsi la vi periller,

Moy meismes a appareiller

La pris ; a clous et a mortel

Rejoing les ais et fort martel ;

Mousse vais cueillant sus les roches,

Es fentes la fiche, a grans troches,

Tant qu'estanche la feis assez

Et rejoigny les bors cassez ${ }^{23}$.

Ce passage reprend le réseau lexical des célèbres vers du Roman de la Rose, Nature qui «dedanz sa forge, /tourjorz martele, torjorz forge ${ }^{24} »$, ainsi que ceux de la scène finale de la cueillette de la rose ou de la défloration, lorsque l'Amant tente de passer par un sentier étroit et «l'escharpe dehors demeure/o les martelez rebillanz, /qui dehors ierent pendillanz $z^{25} »$. Jean de Meun s'était servi de l'agriculture, du travail des métaux et de la création poétique comme métaphores de la génération, les instruments phalliques utilisés dans ces activités rendant l'analogie plus explicite. Christine-narratrice, devenue « vray homs », s'empare elle aussi d'un marteau et de clous et se hâte de boucher les fissures de l'embarcation. L'image est sans équivoque. À travers sa métamorphose et par l'emploi de ce réseau métaphorique, l'auteur revendique une autorité toute masculine et se place au niveau des clercs écrivains.

Il nous reste à examiner cette mutation dans sa relation avec l'activité de Nature: la métamorphose de Christine implique-t-elle une dénaturation, une contrefaçon de l'œuvre de Nature par Fortune? La métamorphose, nous l'avons vu, est intrinsèquement associée à la possibilité de la pratique littéraire. Le topos du combat entre nature et culture permet à Christine de montrer que, tout en possédant, à l'état latent, des dispositions naturelles à l'écriture, elle n'a pas, en tant que femme, les

\footnotetext{
${ }^{23}$ Ibid., v. 1375-1382, p. 52.

${ }^{24}$ Guillaume de Lorris et Jean de Meun, Le Roman de la Rose [1966-1970], éd. Félix Lecoy, Paris, Champion, coll. « CFMA », 1980, 3 vol., t. II, v. 16013-16014, p. 236.

${ }^{25}$ Ibid., t. III, v. 21618-21620, p. 150.
} 
moyens d'écrire. C'est donc en changeant de sexe qu'elle devient écrivain. Il faut néanmoins bien reconnaître que ce changement relève du domaine de la contingence. La poétesse exposera cette idée plus clairement dans son Livre de l'advision, en donnant la parole à Philosophie : «[...] et fust mesmes converti ton corps faible et femmenin en homme pour estre transmuee de condicion $^{26}$ ». Si, d'une part, les caractéristiques contrôlées par Nature appartiennent à l'essence humaine, d'autre part, celles sur lesquelles Fortune a un pouvoir ne peuvent être qu'accidentelles, pour ce que Fortune est une puissance qui régit le hasard.

Dans l'article mentionné plus haut, Jeffrey Richards a analysé la Mutacion de Fortune comme la réfutation directe de l'essentialisme philosophique :

Significantly, her gender was changed, but not her identity. By presenting her metamorphosis as she does, as an act of Fortune that preserves her earlier memory and identity as a woman in the body of a man, Christine radically departs from the essentialist thomist tradition. The stance that Christine takes here also represents an important refinement of her initial position in the Epistre au Dieu d'Amours because her new identity reflects the sum total of the very conditions of her life ${ }^{27}$.

En prenant donc du recul par rapport à la conception qu'elle avait défendue dans ses premiers travaux, à savoir la spécificité de la nature féminine, Christine considère désormais que les différences entre les genres féminin et masculin ne sont pas inhérentes à leur être, mais qu'elles

${ }^{26}$ Christine de Pizan, Le Livre de l'advision..., éd. cit., partie III, chap. XXI, p. 129.

${ }^{27}$ Earl Jeffrey Richards, «Rejecting Essentialism», art. cit., p. 110. «De manière significative, son sexe a été changé, mais pas son identité. En présentant sa métamorphose comme elle le fait, comme un acte de Fortune qui préserve sa mémoire et son identité en tant que femme dans le corps d'un homme, Christine s'écarte radicalement de la tradition thomiste essentialiste. La position qu'elle prend ici représente également un perfectionnement important de sa perspective initiale dans l'Epistre au dieu d'Amours, car sa nouvelle identité reflète la somme totale des conditions mêmes de sa vie » (nous traduisons). 
relèvent des expériences et des conditions historiques. Pour ce qui est de l'affirmation du moi poétique, la métamorphose reste une solution provisoire, qui sera abandonnée dans les œuvres postérieures où l'auteur explorera, comme nous le verrons, d'autres images de la naissance et de la maternité.

\section{La Cité des dames : un modèle de naissance sans père}

La Cité des dames, écrite en 1405, témoigne d'un approfondissement de la réflexion de Christine au sujet de l'être et de la nature humaine. Si l'auteur cite le proverbe «ce que Nature donne, nul ne peut $\operatorname{tolir}^{28}$ », elle l'emploie pour réfuter la prémisse misogyne qui voulait que toutes les femmes soient instinctivement disposées aux vices, autrement dit, qu'elles soient toutes des «montre[s] en nature, [...] chose contrefaite et hors de sa propre condicion naturelle ${ }^{29} »$. Plutôt que de renforcer la dichotomie entre hommes et femmes, Christine accuse - dans le texte, c'est la personnification de Raison qui parle - tout comportement vicieux comme étant une dénaturation des dispositions humaines: «la haulteur ou abaissement des gens ne gist mie es corps selon le sexe, mais en la perfection des meurs et des vertus ${ }^{30} »$. Bien que Nature distribue de façon inégale des caractéristiques comme la beauté, l'entendement et la force corporelle, elle sait les compenser afin de garder toujours la mesure : Aristote, le grand philosophe, n'était-il pas laid ? Les femmes ne sont-elles généralement plus faibles physiquement, mais aussi plus vertueuses? La force vient de la vertu, explique Raison dans ce passage qui semble repenser la représentation de la métamorphose :

Si te promets, belle amie, que le grant et fort corps ne fait mie le vertueux et poissant courage, ains

\footnotetext{
${ }^{28}$ Christine de Pizan, La città delle dame, éd. cit., partie I, chap. X, p. 82.

${ }^{29}$ Ibid., partie I, chap. I, p. 44.

${ }^{30}$ Ibid., partie I, chap. IX, p. 80.
} 
vient d'une vigueur vertueuse naturelle qui est don de Dieu, que il concede a la Nature empraindre es unes creatures raisonnables plus que es autres, et est son giste mucié en l'entendement et ou courage et non mie en la force du corps ou des membres ${ }^{31}$.

Dans ce contexte de réhabilitation morale des femmes et de l'affirmation, par Raison, de leur force, de leur sagesse et de leur intelligence, Christine rejette l'artifice du changement de sexe, devenu désormais caricatural ou grotesque. Ainsi, pour dame Raison, les doutes que la narratrice exprime à propos de son identité et de son sexe rappellent l'anecdote d'un fou qui, habillé en femme, finit par croire en être une. Un autre modèle d'autorité se dessine alors dans cette œuvre, un modèle fondé cette fois-ci sur l'affirmation de la condition féminine de l'auteur. À la métaphore de la naissance sans mère, telle que nous l'avons rencontrée chez Machaut, se substitue celle de la production littéraire comme une naissance sans père ou, tout du moins, sans acte sexuel ni fécondation naturelle.

À l'ouverture du texte, Christine reprend et développe une scène qu'elle avait déjà esquissée dans le Dit de la Rose (1402), une scène de survenue de l'inspiration poétique. L'action avance par détours et l'écrivain procède à un travail sophistiqué d'entrecroisement de symboles. Enfermée dans sa cellule, la narratrice s'adonne à son activité de prédilection, la lecture. L'espace clos, associé à la surabondance de livres qui l'entourent, fixe d'emblée l'image de celle qui entretient avec l'étude des rapports boulimiques. À la fin d'une journée de travail intellectuel intense, Christine cherche à se distraire par la lecture d'un livre qui ne lui appartient pas, Les Lamentations de Mathéolus. Une suspension narrative survient alors: la lecture ne reprend que le lendemain; c'est à ce moment que Christine

${ }^{31}$ Ibid., partie I, chap. XIV, p. 102. 
découvre que le livre n'est qu'un condensé d'idées misogynes. Or, cette lecture l'incite à une profonde remise en question : son œuvre, la Cité des dames, naitra de la confrontation des deux regards, de la réfutation du discours de l'autre.

Christine emprunte au vocabulaire de la génération pour décrire son affliction initiale : «Mais la veue de ycellui dit livre, tout soit il de nulle auctorité, ot engendré en moy nouvelle pensee qui fist naistre en mon courage grant admiracion ${ }^{32} »$. Dans un premier temps, la narratrice accepte les conceptions misogynes des auteurs et philosophes, ce qui pousse le désespoir à son paroxysme et la mène à remettre en cause la volonté divine :

Dieux, pourquoy ne me feis tu naistre au monde en masculin sexe, a celle fin que mes inclinacions feussent toutes a te mieulx servir et que je ne errasse en riens et feusse de si grant perfeccion comme homme masle se dit estre ${ }^{33}$ ?

Ce trouble identitaire plonge la narratrice dans une aliénation comparable à celle du fou travesti. Le discours est bien évidemment ironique. Christine de Pizan aime jouer des procédés antiphrastiques, ce sont ici les propos antiféministes, habillement repris, qui frôlent presque l'hérésie. Plus loin, Raison répond aux misogynes qui accusent Nature d'avoir honte de produire des femmes :

Si n'ot pas honte le souverain ouvrier de faire et former corps femenin, et Nature s'hontoyeroit? $\mathrm{Ha}$ ! la somme des folies de ce dire. Voire, et comment fut elle formee? Je ne scay se tu le nottes - elle fu formee a l'image de Dieu ${ }^{34}$.

La femme, créée à l'image de Dieu, est l'égale de l'homme puisque formée de sa côte. Christine opposera pourtant à Ève, femme pécheresse, la

\footnotetext{
${ }^{32}$ Ibid., partie I, chap. I, p. 42.

${ }^{33}$ Ibid., partie I, chap. I, p. 44.

${ }^{34}$ Ibid., partie I, chap. IX, p. 78.
} 
pureté de Marie, habitante et souveraine de sa cité. L'écrivain, qui croit au pouvoir politique des pleurs féminins ${ }^{35}$, s'identifiera dans d'autres textes à la figure maternelle de la Vierge de douleur, la mater dolorosa. Dans les Lamentacions sur les maux de la France (1410), par exemple, elle décrira les larmes qui tombent sur son giron et qui effacent l'écriture ${ }^{36}$.

Au début de la Cité des dames, le passage par un état d'accablement est indispensable à la manifestation de l'inspiration poétique. Ce topos est traditionnellement représenté par l'apparition, d'ordinaire sur le mode du songe, d'une puissance ou figure allégorique. Ici, c'est une trinité féminine qui intervient, composée de trois vertus, Raison, Droiture et Justice :

En celle dolente pensee ainsi que je estoie, la teste baisse comme personne honteuse, les yeulx plains de larmes, tenant ma main soubz ma joe accoudee sus le pommel de ma chayere, soubdainement sus mon giron vi descendre un ray de lumiere si comme se le souleil fust, et je, qui en lieu obscur estoie ou quel, a celle heure, souleil royer ne peust, tressailli ${ }^{37}$.

Jacqueline Cerquiglini-Toulet, qui a donné une analyse très fine de cette scène, attire l'attention sur le tressaillement comme "signe de cette survenue du merveilleux, passage de l'aile du sacré $^{38} »$. La posture de la narratrice, le regard baissé et la «main à la maisselle », est caractéristique

35 « O ! quantes grans graces fist Dieux a femmes pour cause de leurs larmes », ibid., partie I, chap. X, p. 86.

${ }^{36}$ Voir Louise D'Arcens, "Petit Estat Vesval : Christine de Pizan's Grieving Body Politic », dans Healing the Body Politic : Christine de Pizan's Political Philosophy, éd. Karen Green et Constant Mews, Turnhout, Brepols, coll. «Disputatio », n 7, 2005, p. 201-226; Christine de Pizan, «La Lamentation sur les maux de la France », éd. Angus J. Kennedy, dans Mélanges de Langue et Littérature françaises $d u$ MoyenÂge et Renaissance: offerts à M. Charles Foulon, Rennes, Université de Haute-Bretagne, Institut de Français, 1980, 2 vol., t. I, p. 177-185.

${ }^{37}$ Christine de Pizan, La città delle dame, éd. cit., partie I, chap. II, p. 46.

38 Jacqueline Cerquiglini-Toulet, «L'inspiration des poètes lyriques à la fin du Moyen Âge : le cas de Christine de Pizan ", dans L'inspiration. Le souffle créateur dans les arts, littératures et mystiques du Moyen Âge européen et proche-oriental. Colloque international tenu en Sorbonne les 23-24 mai 2002, éd. Claire Kappler et Roger Grozelier, Paris/Budapest/Kinshasa, L’Harmattan, coll. « Kubaba Actes », 8, 2006, p. 291-302, p. 293. 
d'un état mélancolique qui agit comme catalyseur de l'écriture. Cependant, la force de ce passage réside surtout dans la réécriture de la scène d'Annonciation et dans l'assimilation de la figure de la narratrice à celle de la Vierge. Faisant écho à un autre motif très répandu dans l'iconographie chrétienne - l'on pense au célèbre retable de l'Annonciation (1430) de Fra Angelico - Christine décrit ce rayon de lumière qui descend sur son giron, à l'image de celui qui illumina Marie lorsque l'ange Gabriel l'interrompit dans sa lecture. Notons également que cette lumière, qui s'oriente non pas vers la tête mais vers le giron de Christine, contribue à la reconnaissance du texte comme le produit d'une femme écrivain. Le pouvoir de création est un pouvoir de procréation: les livres de Christine sont véritablement enfantés.

La naissance de l'œuvre est un engendrement. Une homologie s'opère entre une série de lieux clos, inviolés: la matrice de la Vierge, la bibliothèque de Christine (son «estude petite») où naît l'œuvre et la Cité des dames elle-même, qu'elle construit, à la fois lieu et livre. Le Christ naît de la voix du Saint-Esprit murmurant à l'oreille de la Vierge, les livres, chez Christine, naissent des voix « series » (calmes) ou fortes des allégories lui donnant l'ordre de composer ${ }^{39}$.

Christine est chargée par les Vertus de bâtir une ville perpétuelle - le mot est important - le refuge des femmes vertueuses. Pour parler de l'écriture, l'auteur reprend le topos du labour ${ }^{40}$, les sillons creusés dans la terre ne sont pourtant pas évoqués en tant que technique de l'agriculture,

${ }^{39}$ Ibid., p. 295.

${ }^{40}$ Dans un passage déjà mentionné du Roman de la Rose, le personnage de Genius, double de Nature, incite les barons à labourer pour assurer leur descendance. Quelques vers plus loin, le parallèle entre l'acte de forger le fer et celui d'écrire se fait de manière explicite : «Arés, por Dieu, baron, arés, /Et vos lignages reparés. /Se ne pensés forment d'arer, /N'est rienz qui les puist reparer. /[...] Ne vos lessiez pas desconfire, /greffes avez, pansez d'escrire. /N'aiez pas les braz anmouflez:/martelez, forgiez et souflez », Guillaume de Lorris et Jean de Meun, Le Roman de la Rose, éd. cit., t. III, v. 19671-19674 et 19763-19766, p. 91 et 94. 
mais plutôt de l'architecture : «Pren la pioche de ton entendement et fouys fort et fais grant fosse tout partout ou tu verras les trasses de ma ligne», commande Raison, et plus loin: «Si prens la truelle de ta plume et t'apprestes de fort maçonner et ouvrer par grant diligence ${ }^{41}$.» Rappelons que la ville est, par opposition à la vie sauvage, le symbole de l'entrée dans la civilisation, dans la société organisée et réglée. Le travail de l'écrivain, comme celui de l'architecte, est un travail de mise en ordre ${ }^{42}$.

Or, si d'une part les créations de Nature sont périssables, si cette puissance doit travailler sans cesse dans sa forge pour lutter contre la mort, Christine, d'autre part, se vante de donner naissance à une œuvre perdurable et définitive :

Et ores est un nouvel royaume de Femenie encommencié, mais trop plus est digne que cellui de jadis, car ne convendra aux dames ycy herbergees aler hors de leur terre pour concevoir ne enfanter nouvelles heritieres pour maintenir leur possession par diverses aages de ligne en ligne, car assez souffira pour toujours, mais de celles que ores y mettrons ${ }^{43}$.

La vertu de ces fondatrices engendra, à elle seule, les nouvelles habitantes de la cité. Avec des fondations et des pierres solides, des arguments bien construits et des exemples édifiants, Christine donne naissance à un monument littéraire en l'honneur des femmes, lieu utopique et parfait dont la continuité sera toujours assurée par la multiplication perpétuelle de la vertu de celles qui y vivront.

${ }^{41}$ Christine de Pizan, La città delle dame, éd. cit., partie I, chap. VIII, p. 64 et chap. XIV, p. 104.

42 À propos de la notion d'ordre et du mot ordonner, «qui résume le geste du $\mathrm{XIV}^{\mathrm{e}}$ siècle en matière de littérature », voir Jacqueline Cerquiglini-Toulet, "Un engin si soutil », op. cit., p. 15.

${ }^{43}$ Christine de Pizan, La città delle dame, éd. cit., partie II, chap. 12, p. 250. 


\section{L'Advision Cristine : de la conception à la gestation}

La reformulation de la métaphore de la forge de Nature, ainsi que la représentation d'une poétique féminine à travers l'image de la gestation, trouvent leur expression la plus aboutie dans Le Livre de l'advision Cristine, écrit la même année que la Cité des dames. Le développement allégorique y est plus complexe, si bien que le texte est précédé d'une glose où l'auteur précise les différents niveaux de signification. La narration s'ouvre sur deux figures mystérieuses que Christine voit en songe. L'une est une forme masculine colossale, dont le ventre environne la Terre et qui porte sur le front l'inscription C.H.A.O.Z. : il s'agit d'une image du monde. À ses côtés, se trouve la figure qui nous intéressera ici, une « grant ombre couronnee de fourme femminine ${ }^{44} »$, à qui incombe la tâche de nourrir le géant :

Environ soy avoit infinis instrumens de divers coins et empraintes, aussi comme sont a Paris molles a faire gauffres et autres outilz semblables. Et comme ceste dame n'eust en soy le vice de paresce, l'occupoit continuellement neccessaire diligence de divers labours, car sanz prendre repos, elle detrempoit mortier qu'elle coaguloit ensemble, en laquelle mixtion metoit fiel, miel, plomb et plume. D'icelle matiere emplissoit buires de diverses façons, lesquelles aprés versoit en petite quantité es dis moles que bien estouppoit et saeloit. Tout ce fait non d'une guise mais en diverses differences, mettoit tout cuire et confire en la gueule dudit grant ymage [...]. La les laissoit jusques a temps convenable, l'un plus que l'autre, selon la difference et la groisseur des outilz. Aprés le temps venu que la saige admenistraresse savoit le terme de la perfection de son oeuvre, elle ouvroit la bouche de cel ymage par tel art qu'elle donnoit lieu de tirer hors les matieres assés cuites et les autres laissoit cuire

${ }^{44}$ Christine de Pizan, Le Livre de l'advision..., éd. cit., partie I, chap. II, p. 13. 
jusques a l'accomplissement de leurs jours. Adont sailloient hors de ces moles petis corps de diverses façons selon les empraintes des instrumens ${ }^{45}$.

On reconnaît facilement Nature au travail, mais sa forge a été remplacée par une cuisine. Les matériaux qui composent les créatures sont une variation des quatre éléments ; leur forme, quant à elle, est donnée par des moules d'aspect et de tailles différentes. Christine recourt à une métaphore de la grossesse, où le four représente l'utérus et le temps de cuisson la période de gestation. Aussi riche en détails soit sa description, il faut dire que la représentation de la matrice comme moule ou comme four, ainsi que le rapprochement entre les processus physiologiques de gestation et de digestion, sont des analogies trouvées fréquemment dans la littérature médicale, scientifique et encyclopédique médiévale ${ }^{46}$; en revanche, le fait que Christine les utilise pour évoquer Nature est à souligner : à nouveau, elle répond à Jean de Meun, et tend à effacer le rôle de l'homme dans la procréation.

Dans l'Advision, Christine décrit sa propre naissance, son «petit corps humains » façonné par Nature, et la façon dont cette puissance verse la pâte dans le moule et y glisse l'âme, venue de Dieu. Comme dans la Mutacion de Fortune et contre l'idée que la femme serait un mâle manqué, l'identité féminine de Christine tient au choix volontaire de la déesse :

${ }^{45}$ Ibid.

${ }^{46}$ Dans le Placides et Timéo, par exemple, daté de la fin du XIII ${ }^{\mathrm{e}}$ siècle, c'est, non pas un moule à gaufres, mais une faisselle à fromage qui représente l'utérus : " Tout aussi est le marris [c'est-à-dire, la matrice] comme une foisselle ou li sans et le sperme est tout mellés et puis en monte une partie clere as mamelles, qui est convertie en lait. De ce qui remaint si est fourmee le char d'unne partie et les os d'autre, et s'efforce l'ame qui y fu mise avec le sperme de soi eslarguir. Et le char et le lait se prent en la fourme de le marris, aussi comme le fronmage en le foisselle, qui prent celle figure comme la faisselle li amenistre. » (Placides et Timéo ou Li secrés as philosophes, éd. Claude Thomasset, Genève, Droz, coll. "Textes littéraires français », 1980, p. 132). Sur la symbolique de la matrice, voir Sylvie Laurent, Naître au Moyen Âge. De la conception à la naissance : la grossesse et l'accouchement (XII $-X V^{e}$ siècle), Paris, Le Léopard d'Or, 1989, p. 99-103. 
«Mais comme le voulsist ainsi celle qui la destrempe avoit faicte, a laquel cause se tient et non au mole, j'aportay sexe femmenin ${ }^{47}$. »

Comme nous l'avons suggéré plus haut, le rôle de la femme dans la génération était, selon saint Thomas, un rôle passif et par conséquent inférieur à celui de l'homme : en tant que père, l'homme partageait avec Dieu un pouvoir actif, alors que celui de la femme était un pouvoir passif ${ }^{48}$. La femme ne serait donc située que du côté de la matière. Or, dans la dernière partie de l'Advision, Christine narre sa rencontre avec Philosophie (aussi appelée Sainte Théologie). Pendant que la narratrice traverse les antichambres de la demeure de cette dame, on lui laisse amasser des richesses et c'est dans son propre giron que Christine accommode ces trésors : «Et je, de ce non reffusente comme convoiteuse d'en enrichir, en la merciant, me baisse pour mon giron en emplir ${ }^{49}$. » Ce faisant, Christine transforme ce giron maternel, puisqu'il est le lieu où ses œuvres futures seront enfantées, en réceptacle des connaissances et de la sagesse. La valeur de ses œuvres, issues de ce lieu qui abrite les savoirs, est ainsi assurée.

Plus loin, dans le récit biographique et dans la complainte que Christine adresse à Philosophie, nous retrouvons le même modèle de l'accouchement comme image de la création poétique :

[...] ainsi voult [Nature] que par l'engendrement d'estude et des choses nasquissent de moy nouvelles lectures. Adonc me dist: «Prens les outilz et fier sur l'enclume de la matiere que je te bailleray si durable que fer : ne feu ne autre chose ne la pourra despecier; si forges choses delictables. Ou temps que tu portoies les enfans en ton ventre, grant douleur a l'enfanter sentoies. Or vueil que de toy naissent nouveaulx volumes,

\footnotetext{
${ }^{47}$ Christine de Pizan, Le Livre de l'advision..., éd. cit., partie I, chap. 3, p. 14.

${ }^{48}$ Prudence Allen, The Concept of Woman. The Aristotelian Revolution, 750 B.C.A.D. 1250, Montréal, Eden Press, 1985, p. 395.

${ }^{49}$ Christine de Pizan, Le Livre de l'advision..., éd. cit., partie III, chap. 1, p. 92.
} 
lesquelz les temps a venir et perpetuelment au monde presenteront ta memoire devant les princes et par l'univers et en toutes places, lesquelz en joie et delit tu enfanteras de ta memoire, non obstant le labour et traveil, lequel tout ainsi comme la femme qui a enfanté, si tost que elle ot le cry de son enfant oublie son mal, oublieras le traveil du labour oyant la voix de tes volumes ». Adonc me pris a forgier choses jolies $[\ldots]^{50}$.

Christine s'interroge sur la mémoire littéraire en associant subtilement les deux paradigmes de la forge et de l'enfantement. L'auteur retient de l'image de la forge la pérennité des matériaux, mais écarte celle de l'accouplement. Christine adapte ainsi la métaphore de la création littéraire à sa propre réalité de femme : ses livres sont ses enfants.

Il faut souligner la singularité de cette réflexion sur la pratique littéraire. Suggérant une analogie entre création poétique et procréation, l'originalité de Christine réside dans la transformation et l'adaptation de ses modèles pour parvenir à une version féminine de la génération, où toute expression érotique est écartée et où seul le rôle de la femme dans la perpétuation humaine est mis en valeur. Comme l'a signalé Sylvia Huot, « la version de Christine minimise le rôle du désir et crée un mouvement conduisant non plus à la possession, mais à la production ${ }^{51} »$. Ce traitement est un indice de la place qu'a désormais le sujet dans l'œuvre. C'est parce que son écriture est personnelle que Christine doit justifier sa féminité et montrer comment on écrit quant on est une femme. Elle s'impose ainsi comme l'un des grands auteurs de cette époque qui affirme la subjectivité littéraire, ou, pour reprendre la formule de Michel Zink, «l'expression littéraire du sujet par lui-même ${ }^{52}$. »

\footnotetext{
${ }^{50}$ Ibid., partie III, chap. 10, p. 110-111.

${ }^{51}$ Sylvia Huot, "Seduction and Sublimation: Christine de Pizan, Jean de Meun, and Dante », Romance Notes, 25, n ${ }^{\circ}$ 3, 1985, p. 361-373, p. 367.

${ }^{52}$ Michel Zink, La Subjectivité littéraire, op. cit., p. 5. 